\title{
Interfacial two-dimensional oxide enhances photocatalytic activity of graphene/titania via electronic structure modification
}

\author{
Dario De Angelis ${ }^{\mathrm{a}, \uparrow}$, Francesco Presel ${ }^{\mathrm{a}, \uparrow, \mathrm{h}}$, Naila Jabeen ${ }^{\mathrm{a}, \mathrm{b}, \mathrm{c}}$, Luca Bignardi $^{\mathrm{d}, \mathrm{i}}$, Daniel Lizzit ${ }^{\mathrm{d}}$, \\ Paolo Lacovig ${ }^{\mathrm{d}}$, Silvano Lizzit ${ }^{\mathrm{d}}$, Tiziano Montini ${ }^{\mathrm{e}}$, Paolo Fornasiero ${ }^{\mathrm{e}}$, Dario Alfề ${ }^{\mathrm{f}}$, Alessandro \\ Baraldi $^{\mathrm{a}, \mathrm{d}, \mathrm{g}, *}$
}

${ }^{\text {a }}$ Department of Physics, University of Trieste, via Valerio 2, 34127 Trieste, Italy.

${ }^{\mathrm{b}}$ Nanosciences \& Catalysis Division, National Centre for Physics, Islamabad 44000, Pakistan.

${ }^{\mathrm{c}}$ International Centre for Theoretical Physics, Strada Costiera 11, 34151 Trieste, Italy.

${ }^{\mathrm{d}}$ Elettra-Sincrotrone Trieste, S. S. 14, km 163.5 in AREA Science Park, 34149 Trieste, Italy.

${ }^{\text {e }}$ Department of Chemistry and Pharmaceutics, University of Trieste, and INSTM, via L. Giorgieri 1, 34127 Trieste, Italy.

${ }^{\mathrm{f}}$ Department of Earth Sciences, Department of Physics and Astronomy, Thomas Young Centre@UCL, London Centre for Nanotechnology, University College London, Gower Street, WC1E 6BT London, United Kingdom.

g IOM-CNR, Laboratorio TASC, S. S. 14 km 163.5 in AREA Science Park, 34149 Trieste, Italy.

${ }^{\dagger}$ These authors contributed equally.

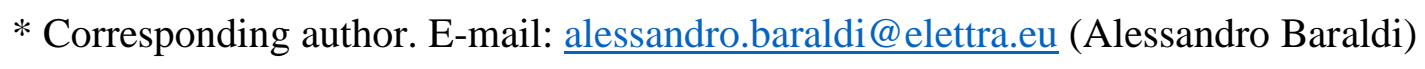

\footnotetext{
${ }^{\text {h }}$ Present address: Department of Physics, Technical University of Denmark, 2800 Kgs. Lyngby, Denmark.
}

${ }^{\text {i }}$ Present address: Department of Physics, University of Trieste, via Valerio 2, 34127 Trieste, Italy. 


\begin{abstract}
The photocatalytic activity of titania nanoparticles deposited on epitaxial graphene is proven to be significantly affected by the substrate on which graphene is supported. In particular, it has been revealed that the addition of a two-dimensional $\mathrm{TiO}_{1.5}$ layer sandwiched between graphene and the supporting metal induces a p-doping of graphene itself and a consistent shift in the Ti $d$ states. These modifications in the electronic structure are compatible with the reduction of the probability of charge carrier recombination and enhance the photocatalytic activity of the heterostructure. This is indicative of the capital role played by the interfacial thin oxide films in fine-tuning the properties of heterostructures based on graphene and pave the way to new combinations of graphene/oxides for photocatalysis-oriented applications.
\end{abstract}

\title{
1. Introduction
}

Carbonaceous materials have been widely employed as fundamental building blocks for heterostructures with catalysis- and energy-oriented applications [1-5]. In particular, Graphene (Gr), owing to its unique electronic and transport properties, is a very good candidate to improve the efficiency of photo-absorbers in photocatalysis and photo-electrocatalysis, which have been targeted as ground stones towards a sustainable energy production [6]. Since the pioneering work of Williams et al. [7], who developed the first titania-graphene nanocomposites, large efforts have been devoted to employ graphene, also in its $3 \mathrm{D}$ form [8], in combination with oxides $\left(\mathrm{WO}_{3}[9,10]\right.$, $\mathrm{ZnO}[11])$ or with other semiconductors $\left(\mathrm{C}_{3} \mathrm{~N}_{4}[12]\right.$, CdS [13]). Graphene, has been used to reduce the electron-hole (e-h) recombination rate, to modify the band alignment, to reduce overpotentials, and to reduce the band gap, thus allowing electrons to be excited into the conduction band by visible light [14]. The latter process can occur following two pathways, either an electron excitation from the valence to the conduction band of $\mathrm{Gr}$ followed by transfer of the electron into the semiconductor unoccupied states, or a direct excitation from occupied Gr states to the absorber's conduction band. Experimental results have revealed that the combination of oxides with Gr can 
improve the degradation of organic molecules [15], extend the light absorption range and enhance the charge separation properties [16 - 18], not only when using oxides in form of thin films or nanoparticles (NPs), but also in other forms such as nanowires [19] and nanotubes [20]. It is important to highlight that most of these results have been obtained using Gr-oxide (GO) or reduced Gr oxide (RGO) [21], which are characterized by poorer transport properties than high-quality epitaxial Gr. Moreover, the presence of structural defects and functional groups in GO and RGO, are known to be centers of e-h recombination, thus lowering the photocatalysis efficiency. This issue strongly limits the photocatalytic enhancement, which is known to be directly related to the charge-separation efficiency [22 - 25], achieved when coupling RGO with semiconductors, to the extent that it has been suggested that GO cannot provide truly new insight into the fabrication of high-performance photocatalysts, in particular when its effect is compared to other carbon-based materials such as carbon nanotubes, fullerenes and activated carbon [26], and points out the importance of using Gr of high structural quality instead.

In this respect, chemical vapor deposition on metal surfaces has proven to be effective for the synthesis of large Gr flakes with a low density of vacancies, domain boundaries and impurities [27]. Moreover, depending on the substrate of choice, this method allows the tuning of the interaction strength between Gr and the metal substrate [28]. In particular, the supporting substrate plays a fundamental role on the degree of Gr doping and therefore represents an opportunity to adjust the properties of Gr-titania hybrid materials by modifying its structure and composition. A powerful strategy to achieve this goal is the intercalation at the Gr-metal interface of any light atoms [2931], molecules [32, 33], alkali- [34], noble- [35] or transition-metals [36] and oxides [37, 38].

Herein, we discuss the growth, the structural/electronic characterization and the photocatalytic activity trends of a novel nano-architecture, which has been designed with the aim to investigate the role of the substrate below Gr on the electronic band structure and alignment of the supported photoabsorbers. By using Gr grown on $\operatorname{Ir}(111)$, as a prototype of quasi free-standing system, we 
have prepared a novel interface consisting of $\mathrm{TiO}_{2}$ NPs supported by a layered structure based on high-quality epitaxial monolayer Gr, two-dimensional titanium-oxide and metal.

In order to disentangle the role of $\mathrm{Gr}$ and its doping level, we synthesized and characterized several nanoarchitectures, where titania was supported on differently doped Gr, as well as a control system without Gr supporting the titania. In particular, we prepared the three systems shown in the bottom part of Figure 1, corresponding to $\mathrm{TiO}_{2}$ supported on (i) a bare $\operatorname{Ir}(111)$ surface (TM, Figure 1d), (ii) Ir-supported Gr (TGM, Figure 1e) and (iii) titanium-oxide-supported Gr (TGTM, Figure 1f).

Lastly, the effects of tuning the electronic structure of $\mathrm{TiO}_{2}$ nanoparticles on its catalytic activity have been investigated by using our system as a model catalyst for the hydrogen evolution reaction (HER). Our experiment shows that the doping of Gr greatly affects the HER reaction rate.

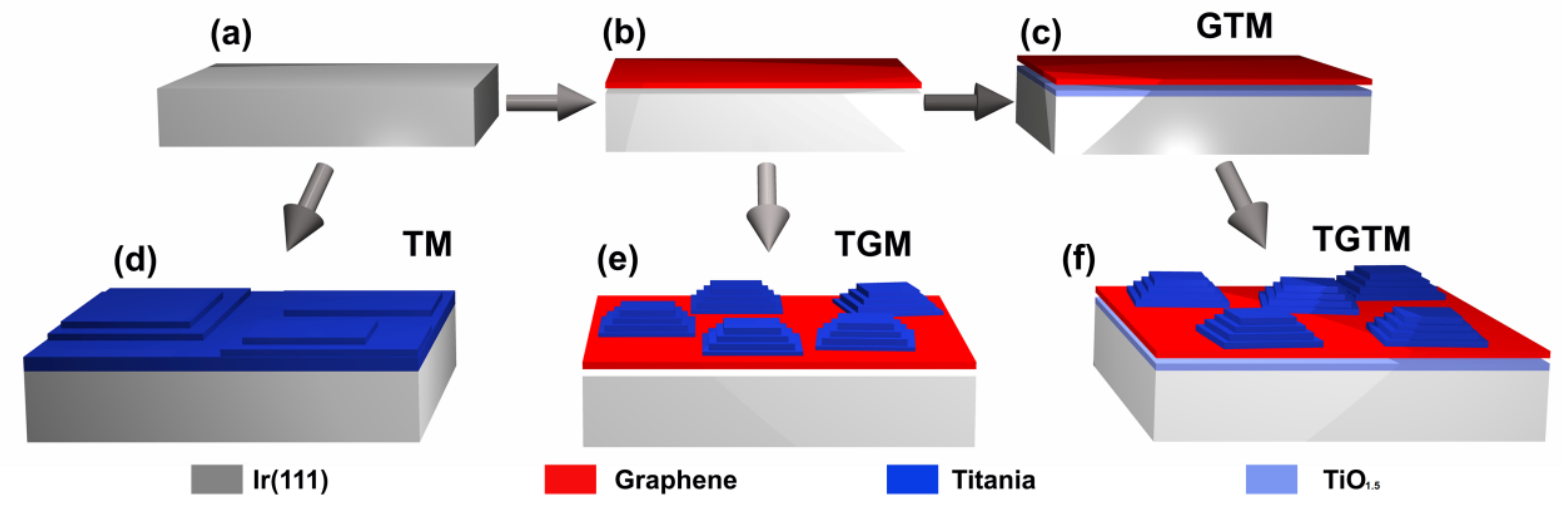

Figure 1. Schematic illustration of the systems prepared in the present work. a) Clean $\operatorname{Ir}(111)$ surface (M); b) Graphene grown on $\operatorname{Ir}(111)$ by means of $\mathrm{C}_{2} \mathrm{H}_{4}$ chemical vapor deposition (GM); c) Graphene on a $\mathrm{TiO}_{1.5}$ interface layer obtained by $\mathrm{Ti}$ intercalation and oxidation $\left.(\mathrm{GTM}) ; \mathrm{d}\right) \mathrm{TiO}_{2}$ grown on $\operatorname{Ir}(111)(\mathrm{TM})$; e) $\mathrm{TiO}_{2}$ nanoparticles grown on $\mathrm{Gr} / \mathrm{Ir}(111)$ (TGM) and $\left.\mathrm{f}\right) \mathrm{TiO}_{2}$ nanoparticles grown on $\mathrm{Gr} / \mathrm{TiO}{ }_{1.5} / \mathrm{Ir}(111)$ (TGTM).

The graphene-based layered nano-architectures we describe could be inspiration for the design of new heterostructures based on ultra-thin surface-oxides [39, 40], opening new pathways for the improvement of energy-based applications of Gr [41] and aiming for a novel generation of photocatalytic materials, both for individual use or in tandem cells. 


\section{Results and discussion}

The preparation stages of the nanostructures are depicted in Figure 1. The Gr/Ir interface (Figure 1b) was obtained by growing single layer Gr on the clean metal surface (Figure 1a) through a wellestablished synthesis method $[42,43]$. It is important to highlight that while the growth parameters (such as substrate temperature and precursor gas pressure) are specific to the Ir substrate chosen for our model system, high quality single layer Gr can be likewise grown on a wide variety of metal surfaces, including common materials such as $\mathrm{Cu}$ and $\mathrm{Ni}$ [28]. Eventually, the Gr/oxide interface (GTM, Figure 1c) was obtained in two steps by intercalation of 0.5 monolayers (ML) of Ti at the Gr/metal interface, followed by oxidation [33,36], as described in the Methods section. As we will show further in the paper, this intercalated oxide was revealed to be a $2 \mathrm{D} \mathrm{TiO}_{1.5}$ layer. Again, this procedure is not specific to the substrate used, but can be applied to a wide variety of $\mathrm{Gr} / \mathrm{metal}$ interfaces [36].

The morphology of $\mathrm{TiO}_{2}$ grown on $\mathrm{Gr} / \mathrm{TiO}_{1.5} / \mathrm{Ir}$ was characterized by scanning tunneling microscopy (STM) (Figure 2a and b), which shows that the surface is homogeneously covered by round-shaped NPs, separated by regions of bare Gr. The average surface corrugation is about $10 \AA$, as illustrated by the scan line profile reported in Figure $2 b$, and it is comparable to the value found for $\mathrm{TiO}_{2}$ NPs grown on $\mathrm{Gr} / \mathrm{Ru}(0001)$ [44]. The size distribution of the particles (Figure 2c), which ranges from 3 to $30 \mathrm{~nm}$, is peaked at $9 \mathrm{~nm}$, comparable to the value found for metallic Ti NPs grown on Gr [45]. A similar distribution of the NPs' sizes was also found for the TGM system, indicating that the presence of the two-dimensional $\mathrm{TiO}_{1.5}$ interfacial oxide layer does not affect the size and distribution of the Gr-supported NPs. In order to have a reference sample to assess the role of graphene in the heterostructure, we have also deposited $\mathrm{TiO}_{2}$ directly on $\operatorname{Ir}(111)$ (TM system, figure 1d) with the same method used for the NPs growth on TGM and TGTM structures. After Ti 
was deposited and oxidized on clean $\operatorname{Ir}(111)$, we could determine by XPS investigation that a $\mathrm{TiO}_{2}$ layer was formed.
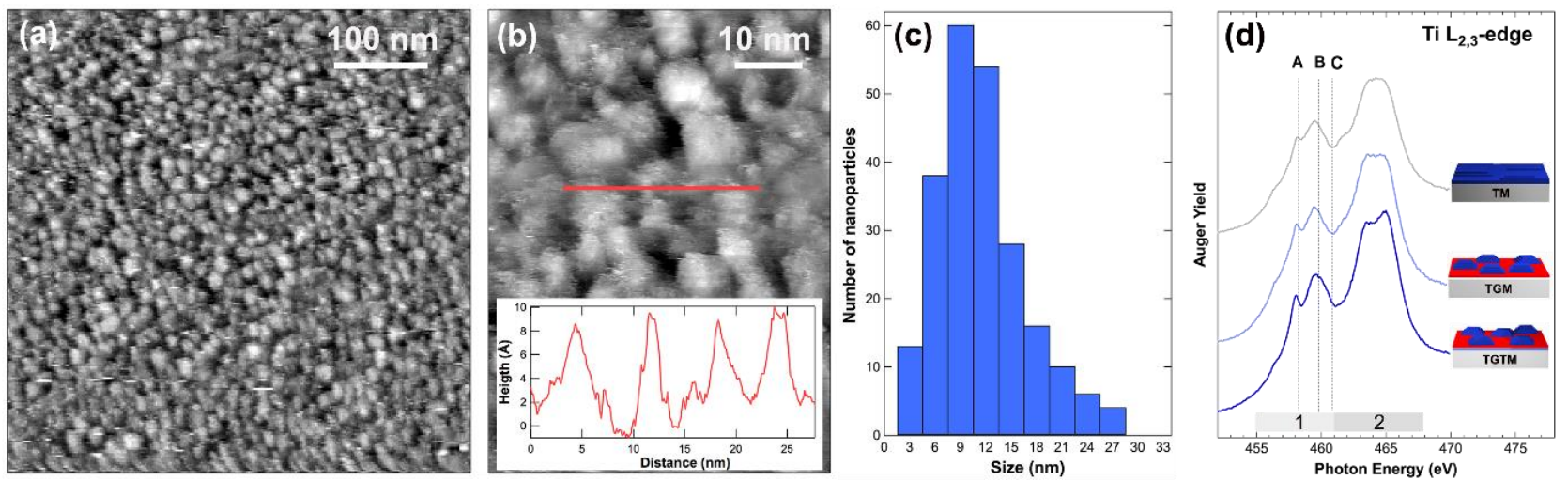

Figure 2. Structural characterization of selected systems. a) STM images of TGTM, $500 \times 500 \mathrm{~nm}^{2}$; b) STM images of TGTM, $100 \times 50 \mathrm{~nm}^{2}$, bias $=2.1 \mathrm{~V}$, current $=0.42 \mathrm{nA}$ ). The inset shows the height profile measured along the red line; c) Size distribution of NPs obtained from a); d) NEXAFS spectra measured at the $\mathrm{Ti}_{1} \mathrm{~L}_{3}$ and $\mathrm{L}_{2}$ absorption edges for the TM, TGM and TGTM nano-architectures. The energies at which the most prominent features A, B and C are usually found in the spectra of bulk titania samples are indicated with dotted lines.

The local crystal structure of the Ti dioxide NPs on top of graphene was investigated by analyzing near-edge X-ray absorption fine structure (NEXAFS) spectra at the $\mathrm{Ti} \mathrm{L}_{3}(455-461 \mathrm{eV}$-region 1) and $\mathrm{L}_{2}(461$ - $468 \mathrm{eV}$-region 2) thresholds (Figure 2d). Both regions show an intense and structured pre-edge feature. The fine structure is due to the splitting of the Ti $d^{*}$ bands due to the crystal symmetry. For this reason, the energy value of the maxima is a fingerprint of the various existing $\mathrm{TiO}_{2}$ crystal structures, thus allowing for example to distinguish the rutile and the anatase phases in titania [46]. More specifically, in our case the $\mathrm{L}_{3}$ maxima are found at $458.0 \mathrm{eV}$ (A) and $459.8 \mathrm{eV}$ (B), which indicates that the local crystal structure in our $\mathrm{TiO}_{2} \mathrm{NPs}$ is anatase. Interestingly, $\mathrm{TiO}_{2}$ NPs are commonly of the anatase form [47,48], which is generally considered a better photocatalyst than rutile, the most stable polymorph of $\mathrm{TiO}_{2}$, because of its longer carrier lifetime, longer e-h diffusion length, higher surface area and higher carrier mobility. 
A very important characteristic of titania NPs is their oxidation state, which is related to the density of $\mathrm{O}$ vacancies, which have been proven to play an essential role in determining their photocatalytic

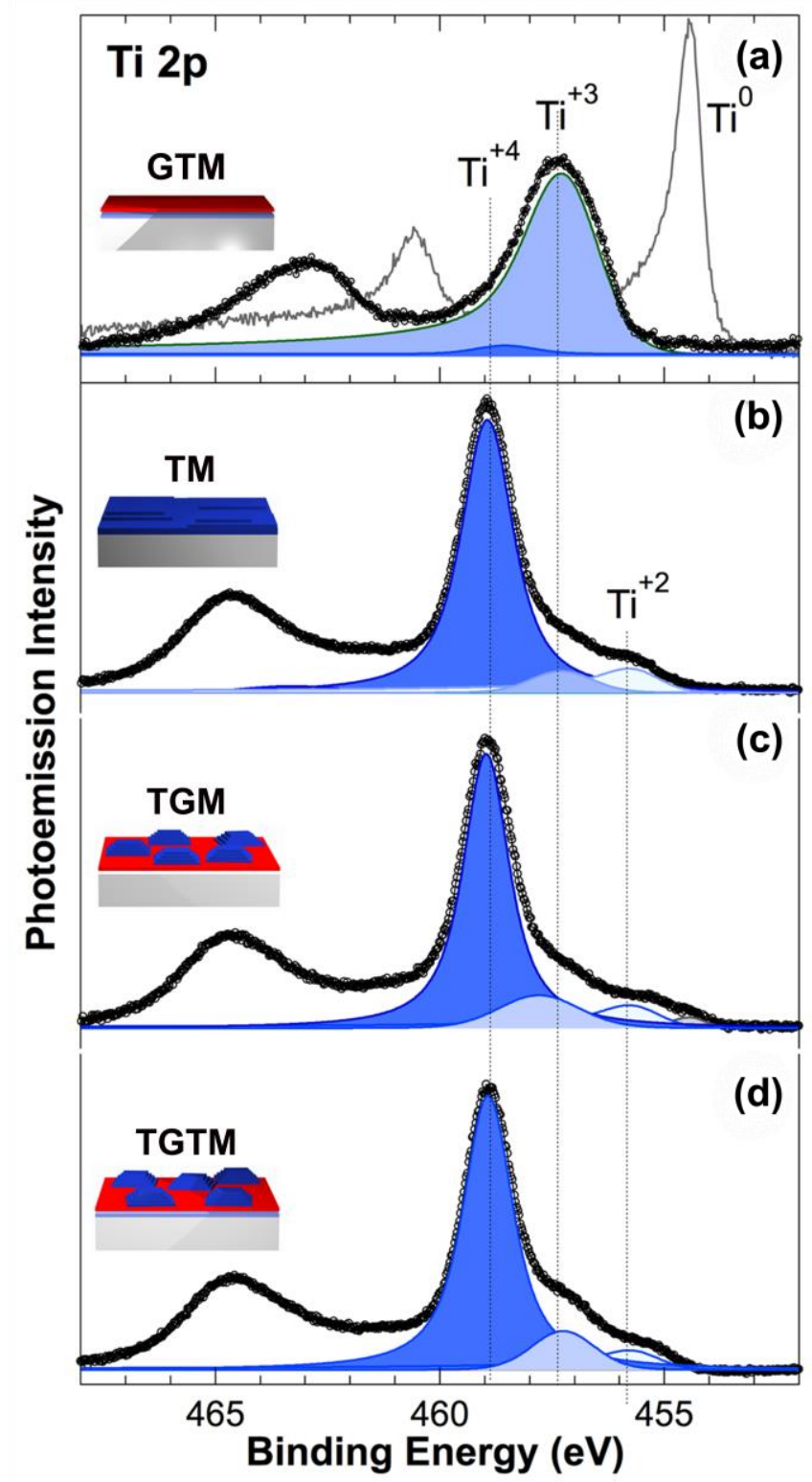

activity. In order to assess the vacancy density, we have identified the oxidation states of titanium in each nanoarchitecture by a quantitative analysis of the high-resolution (HR) X-ray photoelectron spectroscopy (XPS) spectra of the Ti $2 p$ levels (shown in Figure 3).

Figure 3. $\mathrm{Ti} 2 p$ photoemission spectra. $2 p_{3 / 2}$ spectra together with deconvoluted spectral components are reported for (a) GTM, (b) TM, (c) TGM and (d) TGTM nano-architectures (hv = $550 \mathrm{eV}$ ). The spectrum of the as-deposited metallic titanium, before oxidation, is shown in the top graph for comparison (grey curve). 
The Ti $2 p$ spectrum corresponding to the titanium intercalated layer is shown in Figure $3 a$. The $2 p_{3 / 2}$ spectral region is characterized by a main peak a binding energy (BE) of about $457 \mathrm{eV}$ and an additional feature at about $459 \mathrm{eV}$, which can be associated to $\mathrm{Ti}^{3+}$ and $\mathrm{Ti}^{4+}$ oxidation states, respectively [49]. The Ti $2 p$ spectrum corresponding to the intercalated metallic Ti layer before oxidation, characterized by the $\mathrm{Ti}^{0}$ component at $454.35 \mathrm{eV}$, is also shown for comparison (grey spectrum in the top panel of Figure 3). In the GTM architecture, the component with the highest spectral weight is the one originated by $\mathrm{Ti}^{3+}$. The quantitative analysis of the $\mathrm{Ti} 2 p$ core level indicates that oxygen to $\mathrm{Ti}$ atoms are in a ratio of about $2: 3$ for this architecture. Interestingly, this corresponds to the $\mathrm{TiO}_{1.5}$ layer that was observed for a $(2 \times 2)$ long range order structure formed by 2D titanium oxide on $\operatorname{Pt}(111)$ at $670 \mathrm{~K}$ [50], i.e. the same temperature we have employed when oxidizing the intercalated Ti. The low energy electron diffraction pattern we acquired on the GTM interface shows indeed a (2x2) pattern (see supplementary data). The presence of a small amount of Ti atoms in the higher +4 oxidation state might be due to local defects such as additional $\mathrm{O}$ atoms at the interface between this layer and iridium, or a local bilayer structure with the stoichiometry of titania.

Following this spectroscopic characterization of the $\mathrm{Gr} / \mathrm{TiO}_{1.5} / \mathrm{Ir}$ interface, we evaluated the $\mathrm{Ti}$ oxidation state in titania for the TM, TGM and TGTM architectures, whose Ti $2 p$ spectra are shown in Figure 3(b, c, d). In TM, TGM and TGTM the main peak at about $459 \mathrm{eV}$ is attributed to Ti atoms in +4 oxidation state. Additional low-intensity components are observed at lower BE and are a fingerprint of the presence of oxygen vacancies. In each of the three systems, the relative spectral weight of the $\mathrm{Ti}^{4+}$ species is between 75 and $82 \%$. It is important to remark that the Ti $2 \mathrm{p}$ spectrum for the TGTM structure is dominated by the signal coming from the supported titania NPs, whose shape and position is very similar to those observed for the TGM. The component associated with the interfacial oxide layer (i.e. the only one observed for the GTM system) is also present but very 
attenuated, because of the inelastic mean free path of the electrons emitted from the interfacial $\mathrm{TiO}_{1.5}$ layer, which is buried under graphene and the topmost titania.

XPS outcomes were combined with the results obtained from resonant photoemission spectroscopy (RESPES) experiments (see Supporting Information), in order to extract information about the density of oxygen defects in $\mathrm{TiO}_{2}$ in the TM, TGM and TGTM nanoarchitectures. In resonant conditions, a spectral feature associated to the presence of oxygen vacancies is easily measurable at about $1.1 \mathrm{eV} \mathrm{BE} \mathrm{[51].} \mathrm{Its} \mathrm{intensity} \mathrm{difference} \mathrm{between} \mathrm{TGM} \mathrm{and} \mathrm{TGTM} \mathrm{is} \mathrm{less} \mathrm{than} \mathrm{1 \% ,} \mathrm{showing}$ that the particles grown on the two substrates are indistinguishable from this point of view. For the TM interface, the photoemission signal is generally higher, but the relative intensity of the $1 \mathrm{eV}$ feature does not show significant differences, implying that the density of $O$ vacancies does not significantly differ in the three architectures. RESPES measurements were also used to extract the value of the band gap of titania, using the method proposed by Das et al. [52] The value of $4.3 \mathrm{eV}$, which is overestimated when compared to the one obtained using other methods, mainly due to the errors introduced by Koopmans' approximation and to final state effects, is in good agreement with the one we measured for bulk anatase using the same method. This suggests that the size of the NPs is large enough to consider their band-gap the same as bulk-like anatase titania.

HR-XPS experiments returned also important information about the charge transfer occurring between Gr and both its substrate and the NPs, whose composition influences the doping level of Gr and affects the BE value of the C $1 s$ core level peak [53]. Figure 4a shows the C $1 s$ core level spectra of $\mathrm{Gr} / \mathrm{Ir}$ and $\mathrm{Gr} / \mathrm{TiO}_{1.5} / \mathrm{Ir}$ before and after the growth of $\mathrm{TiO}_{2}$ NPs. In particular, the C $1 s$ observed for $\mathrm{Gr} / \mathrm{Ir}$ is centered at $284.12 \mathrm{eV}$, indicating a slight p-doping [54,55]. The growth of titania NPs on Gr/Ir (TGM spectrum) results in a shift towards higher BE. The growth of an oxide layer on top of $\mathrm{Gr}$ is expected to result in an increase of the $\mathrm{C} 1 s \mathrm{BE}$ because of a shift in the Fermi level, which causes a n-doping of the Gr layer. This similar to the results observed for the case of yttria-covered epitaxial Gr grown on $\mathrm{Pt}(111)$ [56]. The intercalation of a 0.5 ML of Ti below Gr produced the same type of doping, but even to a higher extent, thus resulting in a $\mathrm{C} 1 \mathrm{~s}$ component 
shifted to higher BE (284.7 eV), as observed for Ti deposited on SiC-supported $\mathrm{Gr}$ [57]. A corrugation induced in $\mathrm{Gr}$ in strongly interacting systems by the lattice mismatch between $\mathrm{Gr}$ and the metal substrate accounts for the double C $1 s$ component detected [36,58,59]. However, after oxidizing the intercalated $\mathrm{Ti}$ layer, the $\mathrm{C} 1 \mathrm{~s}$ peak shifts to an even lower binding energy with respect to $\mathrm{Gr} / \mathrm{Ir}$ (about $-0.50 \mathrm{eV}$ ), which is the fingerprint, in this configuration, of strong p-doping of the Gr layer. The presence of interfacial oxide layers, e.g. alumina [37], in between $\mathrm{Gr}$ and the supporting metal substrate, also resulted in a considerable p-doping, although in these cases the core level shifts were smaller. Last, the deposition of titania on top of $\mathrm{Gr} / \mathrm{TiO}_{1.5} / \mathrm{Ir}$ nanoarchitecture (TGTM spectrum) shifted again the C $1 s$ BE towards higher values, only partially compensating the effect of p-doping due to the presence of the interfacial oxide $(\mathrm{C} 1 s \mathrm{BE}=283.95 \mathrm{eV})$.

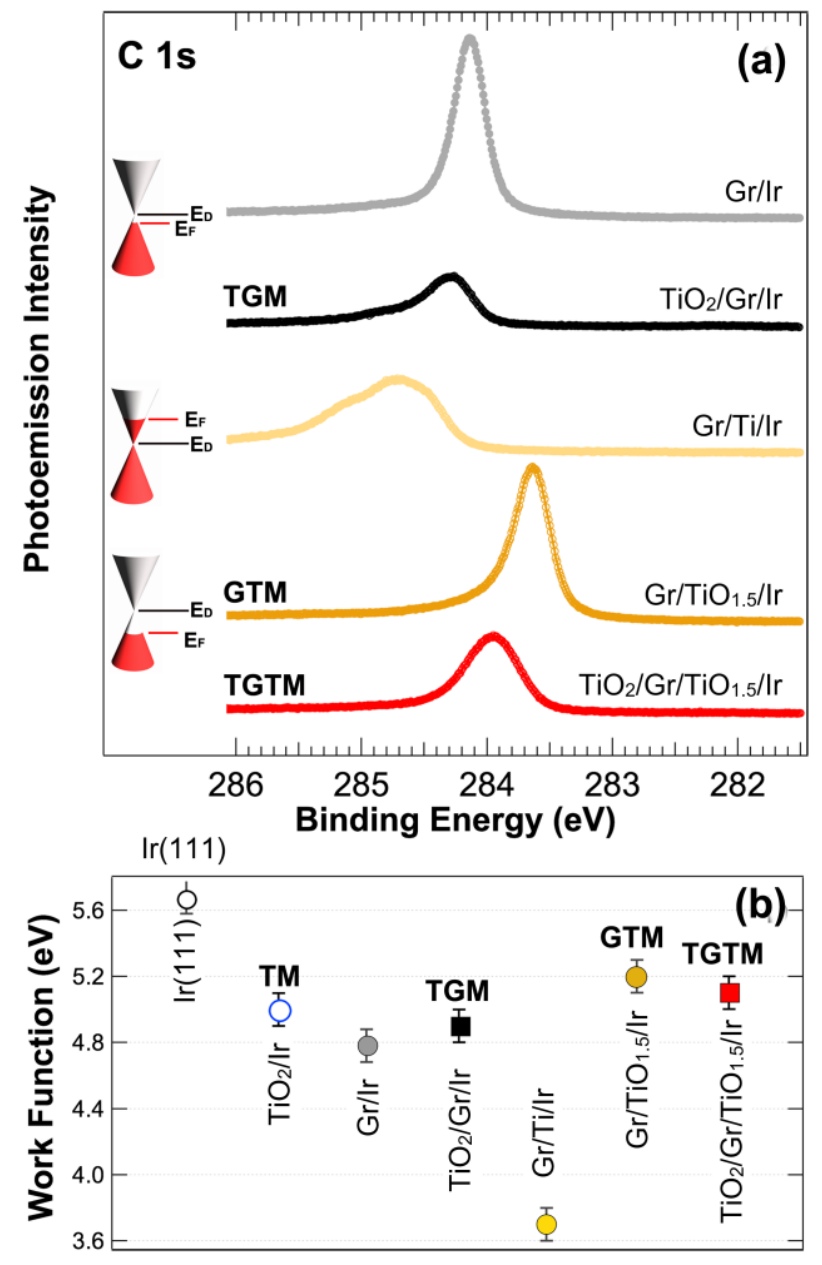

Figure 4. Charge redistribution in the different nano-architectures. a) $\mathrm{C} 1 s$ core level photoemission spectra measured on selected systems $(\mathrm{h} v=400 \mathrm{eV})$. The approximate position of the Fermi level 
$\mathrm{E}_{\mathrm{F}}$ with respect to the Dirac cone $\mathrm{E}_{\mathrm{D}}$ is also reported; b) Comparison of the work function measured on the different nano-architectures.

These differences in the Gr substrate doping are expected to affect the $\mathrm{TiO}_{2}$ NPs by modifying their electronic level occupation, and thereby their work function (WF). Therefore, we have assessed how the presence of Gr and its substrate affect the WF of the different nanoarchitectures. Figure $4 \mathrm{~b}$ displays the WF of the three substrates, before (circles) and after (squares) the growth of $\mathrm{TiO}_{2} \mathrm{NPs}$. The values of WF for the TM, TGM and TGTM are close to the value of $5.1 \mathrm{eV}$ reported for bulk anatase, albeit there are differences of a few hundred meV among them. The WF of the Gr/Ir (grey circle) and $\mathrm{Gr} / \mathrm{TiO}_{1.5} / \mathrm{Ir}$ (orange circle) clearly reflects the shift observed in the $\mathrm{C} 1 s$ core level, as already reported [53]: the WF of the former is indeed lower than that of the latter by about 400 $\mathrm{meV}$. It is interesting to highlight that the $\mathrm{WF}$ of the $\mathrm{Gr} / \mathrm{Ir}$ and $\mathrm{G} / \mathrm{TiO}{ }_{1.5} / \mathrm{Ir}$ structures also affects that one of the NPs supported on them. In particular, the WF measured for the TGM and TGTM returns a value that is in between that one of bulk $\mathrm{TiO}_{2}$ and that of the respective substrate, indicating a charge transfer to or from the NPs depending on the degree of Gr doping.

After this characterization, we qualitatively tested the photocatalytic activity of the different architectures for a test reaction, i.e. the hydrogen evolution half-reaction. To this purpose, the samples were immersed in a 1:1 water/methanol solution and exposed to the radiation of a solar simulator. Methanol is conventionally used as sacrificial reagent, that can interact with the photogenerated holes and be oxidized more easily with respect to water, avoiding the partial back recombination of $\mathrm{H}_{2}$ and $\mathrm{O}_{2}$ formed during pure water splitting. These measurements are meant to show qualitative trends between our systems, while an evaluation of the actual performance of these catalysts is outside the scope of our experiment. A reference measurement was taken in the same conditions on a $\mathrm{Gr} / \mathrm{Titania}$ catalyst obtained combining commercial $\mathrm{GO}$ and $\mathrm{TiO}_{2}$ nanoparticles (see Supporting Information for details). The activity of the nano-architectures was evaluated by measuring the amount of $\mathrm{H}_{2}$ gas produced in 20 hours. The results of the measurements of the TM, TGM and TGTM systems are reported in Table 1. It is interesting to note that a significant effect is 
observed when comparing the activity of TGM and TGTM. The latter system has an activity that is much larger than the former, thus proving that the presence of a two-dimensional $\mathrm{TiO}_{1.5}$ oxide between the metal substrate and Gr plays a vital role in driving the photocatalytic efficiency of the supported catalyst.

Table 1. Photocatalytic measurements. Hydrogen production, expressed in $\left[\mathrm{mol} \mathrm{H}_{2} / \mathrm{mol} \mathrm{TiO}_{2}\right]$, from a water/methanol 1:1 solution in 20 hours under simulated solar illumination for the TM, TGM and TGTM nano-architectures, normalized by the quantity of titania.

\begin{tabular}{llll}
\hline System & TM & TGM & TGTM \\
\hline $\mathrm{H}_{2}$ production $\left[\mathrm{mol} \mathrm{H}_{2} / \mathrm{mol} \mathrm{TiO}_{2}\right]$ & $0.035 \pm 0.009$ & $0.103 \pm 0.008$ & $0.835 \pm 0.005$ \\
\hline
\end{tabular}

To shed light on the origin of these large differences among the various nano-architectures and to understand the fundamental role of the $\mathrm{TiO}_{1.5}$ surface oxide at the interface, we performed density functional theory (DFT) calculations for the TGM and TGTM architectures. The systems were modelled with a slab of 4 layers of Ir in a $10 \times 10$ hexagonal supercell. The bottom two layers of Ir were kept frozen at their bulk geometry, with a lattice parameter of $2.74 \AA$. A $\mathrm{TiO}_{1.5}$ layer was initially placed on top of Ir for the case of the TGTM system, using the geometry experimentally observed for a monolayer of oxidized Ti on the $\mathrm{Pt}(111)$ surface having this stoichiometry. In both TGM and TGTM systems, $(11 \times 11)$ unit cells of graphene were superimposed. This definition of the supercell was able to describe the $(2 \times 2)$ periodicity of the $\mathrm{TiO}_{1.5}$ layer below graphene. This $(11 \times 11)$ periodicity for graphene, which corresponds to a $(10 \times 10)$ Ir supercell, does not exactly reproduce the experimentally observed one, but it results in a graphene lattice strain that is less than 1\%. Eventually, titania layers in the anatase configuration with (001) and (101) surface terminations were placed on top of Gr, with a thickness of 7.94 and $9.70 \AA$, respectively, i.e. very similar to the 
one measured by STM. The total number of atoms considered for the TGTM system was 1397 (400 Ir, $260 \mathrm{Ti}, 242 \mathrm{C}$ and $495 \mathrm{O})$.

Interestingly, the (101) surface of anatase (see Figure 5a and b), which is known to be the most efficient in the reduction reactions [60] because of the electron trapping at the aqueous-surface interface [61], resulted to be energetically more stable by $35 \mathrm{eV}$, in agreement with the Wulff construction [62]. The (101) titania anatase surface exhibits the typical sawtooth-like corrugation with 6- and 5-fold coordinated $\mathrm{Ti}$, and 3- and 2-fold coordinated $\mathrm{O}$ atoms [59]. On the contrary, the finite thickness of the (100) slab results in a large deformation of the anatase structure (see Supporting Information), indicating that the tendency of this surface to reconstruct [63] is also present in the case of a thin film supported by $\mathrm{Gr}$. The introduction of a $\mathrm{TiO}_{1.5}$ surface-oxide in between $\mathrm{Gr}$ and the metal substrate almost entirely removes the $0.32 \AA$ corrugation of the carbon layer which is present in the TGM structure. For the two layered structures, the titania-Gr distance is not appreciably different (see Figure 5a and b).
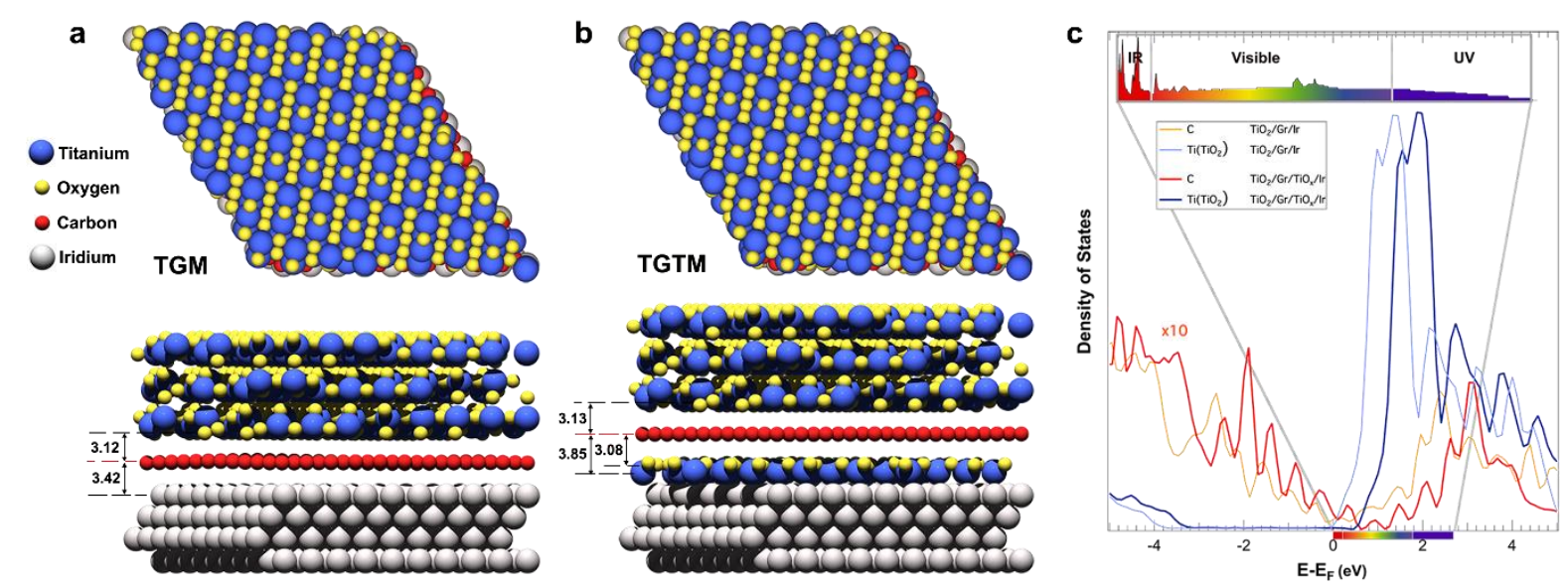

Figure 5. Geometric and electronic structure of the TGM and TGTM nanoarchitectures from DFT calculations. Top and side view of the a) relaxed TGM and b) TGTM supercells; c), calculated partial density of states of TGM (yellow and light blue curves are multiplied by a factor 10) and TGTM (red and blue curves) with (101) surface termination. 
To understand the role of the interfacial $\mathrm{TiO}_{1.5}$ oxide, we computed the density of states (DOS) projected on the carbon atoms and on the titanium atoms of the $\mathrm{TiO}_{2}$-anatase layer. Besides the band gap of $3.2 \mathrm{eV}$, which is in good agreement with the optical gap of anatase measured at $4 \mathrm{~K}$ [64], Figure 5c clearly shows that both the valence and conduction bands of $\mathrm{TiO}_{2}$ are shifted by a similar amount (about $0.5 \mathrm{eV}$ ) to higher energies for the TGTM architecture. Interestingly, the same overall upward shift of $0.5 \mathrm{eV}$ can be appreciated also in the $\pi$ and $\pi^{*}$ bands of $\mathrm{Gr}$ (red curve), as a main perturbation caused by the addition of the $\mathrm{TiO}_{1.5}$ interfacial oxide. This clearly reflects the experimentally observed effect of the Gr doping and work function of $\mathrm{Gr}$ on the work function of the NPs. In particular, because of the Gr p-doping, the bottom of the titania conduction band moves away from the Fermi level, with the important effect of a region just above this level characterized by a zero density of empty states, on the contrary to what is found for the TGM structure where the lower edge of the $\mathrm{Ti} 3 d^{*}$ band lies very close to the Fermi level and the $\mathrm{TiO}_{2}$ is almost degenerate. In addition, the higher separation of these states from the Fermi level in the TGTM system has a dramatic effect on the de-excitation channels available to the electrons excited into them. In particular, one of the most probable decay channels, i.e. a phonon-assisted process, requires a much larger number of electron-phonon scattering events the further the states are from the Fermi level. Therefore, the higher energy of the Ti $3 d^{*}$ conduction band states considerably reduces the e-h pairs recombination in Gr in TGTM when compared with TGM. Further contributions that might explain the enhanced reactivity of the novel nano-architecture can be found in the higher Gr DOS at the Fermi level and in the reduction of the Gr DOS above the Fermi energy. The former increases the number of excited electrons, the latter reduces the possibility of electron excited in the conduction band of Gr to recombine with holes via phonon-assisted processes. All these contributions affecting the excitation and de-excitation channels of e-h pairs allow this p-doped nanostructure to show a dramatically increased performance despite its chemical state and geometrical structure not showing any significant difference. 


\section{Conclusion}

In summary, we have proven that the photocatalytic activity of titania NPs deposited on epitaxial single-layer graphene on $\operatorname{Ir}(111)$ is enhanced when a $2 \mathrm{D} \mathrm{TiO}_{1.5}$ layer is intercalated between graphene and the metallic substrate. This enhancement is ascribed to the different doping level induced in graphene by the $\mathrm{TiO}_{1.5}$ layer and by the shifts of the $\mathrm{Ti} d$ band of the titania NPs.

The change in the electronic structure of the layered material is indicative of a reduced probability of recombination of electrons and holes which are produced in the photoexcitation process. Our results are a proof that the use of 2D materials, and in particular of transfer-free epitaxially grown materials and their heterostructures could be applied for the design of novel energy related materials with greatly improved functionalities.

\section{Methods}

\subsection{Experimental methods}

The $\operatorname{Ir}(111)$ surface was cleaned by repeated cycles of $\mathrm{Ar}^{+}$sputtering and flash annealing to $1400 \mathrm{~K}$, followed by annealing in $\mathrm{O}_{2}$ and in $\mathrm{H}_{2}$ gas [65]. The single-layer $\mathrm{Gr}$ growth on $\operatorname{Ir}(111)$ has been performed by repeated annealing cycles of the sample to $1420 \mathrm{~K}$ while exposing it to up to $3 \times 10^{-7}$ mbar partial pressure of ethylene. We checked the quality of Gr by low-energy electron diffraction and scanning tunneling microscopy, to evaluate its long-range order and density of point-like defects, and high-resolution photoelectron spectroscopy. The titanium oxide intercalation below graphene was achieved with a two-step procedure. Firstly, we exposed the graphene-covered surface to $\mathrm{Ti}$ sublimated from a high-purity filament, while keeping the sample at $670 \mathrm{~K}$. A quantitative XPS measurement confirmed the presence of $0.5 \mathrm{ML}$ of $\mathrm{Ti}$ on the surface, which caused a complete disappearance of the surface component in the $\operatorname{Ir} 4 f_{7 / 2}$ core level. This implies 
that all the atoms in the topmost layer of the Ir surface are in contact with the Ti atoms, as described in Refs. [36] and [66]. Afterwards, we exposed the sample to $5 \times 10^{-3}$ mbar partial pressure of oxygen for one hour, while keeping it at $570 \mathrm{~K}$. We employed a two-step procedure for the NPs growth on graphene, keeping the sample at room temperature and exposing it to Ti atoms first and then to a partial pressure of $1 \times 10^{-6}$ mbar of oxygen for ten minutes. The amount of titanium deposited for each architecture was quantified using different experimental techniques. Quantitative XPS was used to evaluate the surface density of Ti atoms deposited on the substrate. For the TM structure, the thickness of the $\mathrm{TiO}_{2}$ layer was calculated from the attenuation of the photoemission signal of the $\operatorname{Ir} 4 f_{7 / 2}$ core level with respect to the clean Ir surface. The rate of Ti deposition was calibrated by means of a quartz microbalance. Then, the same amount of titania was deposited on $\operatorname{Ir}(111), \mathrm{Gr} / \mathrm{Ir}(111)$ and $\mathrm{Gr} / \mathrm{TiO}_{1.5} / \mathrm{Ir}(111)$. See Supplementary Data for details.

HR-XPS measurements were performed in-situ at the SuperESCA beamline at Elettra. The experimental chamber is equipped with a Phoibos hemispherical electron energy analyzer, provided with a delay line detector. The overall energy resolution was always better than $100 \mathrm{meV}$ for the photon energies and parameters employed. The XPS spectra were acquired by tuning the photon energy in order to have a photoelectron kinetic energy of about $100 \mathrm{eV}$, to enhance surface sensitivity. For each spectrum, the photoemission intensity was normalized to the photon flux and the binding energy scale was aligned to the Fermi level of the iridium substrate. For the fitting procedure of the core levels, a Doniach-Šunjić line profile has been used for each spectral component, convoluted with a Gaussian distribution to account for the experimental, phonon and inhomogeneous broadening [67]. The background was modeled with a polynomial of first order for all spectra, except for those of the Ti $2 p$, where a second order polynomial was used. Resonant Photoelectron Spectroscopy was performed by scanning across the Ti $\mathrm{L}_{3}$-edge - between 452 and $462 \mathrm{eV}$ photon energy - while measuring the valence band spectral region. The photon energy was varied by $0.1 \mathrm{eV}$ at each step and the photon energy calibration was performed by using the second 
order diffraction of the monochromator. The on-resonance photon energy was defined in coincidence to the maximum photoemission intensity in the valence band region at $458.8 \mathrm{eV}$. For the off-resonance a photon energy of $451 \mathrm{eV}$ was selected.

The surface work function was evaluated by measuring the kinetic energy onset of the secondary electrons signal, using a (previously calibrated) photon energy of $140 \mathrm{eV}$. In order to be able to carry out this measurement also for samples having a lower WF than the analyzer, a bias of $-10 \mathrm{~V}$ was applied to the sample in the measurements.

NEXAFS measurements have been performed in Auger yield mode at the SuperESCA beamline, normalizing the intensity to the photon flux for each photon energy. The photon flux has been measured via the total drain current from a gold mesh intercepting the photon beam.

STM measurement have been performed ex-situ at the CoSMoS experimental station of the SuperESCA beamline using a SPECS STM 150 Aarhus equipment. The images reported in this article have been acquired using a tungsten tip, at constant current $(0.47 \mathrm{nA})$ with $2.1 \mathrm{~V}$ bias and at room temperature. The calibration of the perpendicular coordinate has been done by measuring the already known height of the step between iridium surface and Gr. For the image analysis, we used the free open-source software Gwyddion [68]. The statistical analysis on the cluster size has been performed using the specific built-in functions. The photocatalytic activity was evaluated under simulated sunlight irradiation using a solar simulator (LOT-Oriel) equipped with a $150 \mathrm{~W}$ Xe lamp and an atmospheric edge filter to cut-off UV photons below $300 \mathrm{~nm}$. The beam was focused on the sample and the resulting light intensity was $25 \mathrm{~mW} \mathrm{~cm}^{-2}(250-400 \mathrm{~nm}, \mathrm{UV}-\mathrm{A})$ and $180 \mathrm{~mW} \mathrm{~cm}^{-2}$ (400 - $1000 \mathrm{~nm}$, Vis-NIR). The incident illumination power is close to 2 Suns and is representative of a simple but effective solar concentrator. The photocatalytic hydrogen evolution reaction experiments were performed with head spaced vials (total volume $20 \mathrm{~mL}$ ) filled with $12.5 \mathrm{~mL}$ of a water/methanol 1:1 v/v solution. The vial was then sealed using appropriate rubber septa and the air was removed by bubbling Ar for 30 minutes. After this equilibration period, the sample was 
irradiated for 20 hours at room temperature. The analysis of the reaction products was performed injecting $50 \mu \mathrm{L}$ of the gas phase into a gas chromatograph (Agilent 7890), after adding $250 \mu \mathrm{L}$ as internal standard. The Thermal Conductivity Detector (TCD) was used for the quantification of $\mathrm{H}_{2}$, using a MolSIEVE 5A column with Ar as carrier.

\subsection{Theoretical methods}

The calculations have been performed using DFT as implemented in the VASP code [69]. The atomic structure of the studied systems was fully relaxed using the rev-vdw-DF2 functional until the largest residual force was less than $0.015 \mathrm{eV} / \AA$ [70]. We employed the projector augmented method (PAW) [71], using PBE potentials [72], with 9, 4, 4 and 6 electrons in valence for Ir, Ti, C and O, respectively. The plane wave cutoff was set to $400 \mathrm{eV}$, and the relaxations were performed by sampling the Brillouin zone using the $\Gamma$ point only. Although the geometry of the system is accurately described by the rev-vdw-DF2 functional, its electronic structure requires the use of a hybrid functional, as pointed out earlier [73]. To obtain the partial density of states (PDOS) we have therefore performed single point DFT calculations with the HSE06 functional [74], using geometries obtained with the rev-vdw-DF2 functional. Because of the size of the system, each HSE06 calculation required several weeks of running on 768 cores of a Cray-XC30 supercomputer. By contrast, similar calculations with the rev-vdw-DF2 functional only took a few minutes. With our supercell, the PDOS of Gr is poorly described by the $\Gamma$ point only, requiring at least a $3 \times 3$ Monkhorst-Pack k-point grid [75], but the PDOS of Ti is already very accurate with just the $\Gamma$ point.

\section{Acknowledgments}

We are grateful, for computational resources, to ARCHER UK National Supercomputing Service, United Kingdom (NE/M000990/1 and NE/R000425/1), the University College London (UCL) Research Computing, the MMM hub (EP/P020194/1) and Oak Ridge Leadership Computing Facility (DE-AC05-00OR22725). D. De Angelis and F. Presel contributed equally to this work. 


\section{Conflict of Interest}

The authors declare no conflict of interest.

\section{Appendix A. Supplementary Data}

Supplementary data to this article can be found online at

\section{References}

[1] H. Yu, Y. Zhao, C. Zhou, L. Shang, Y. Peng, Y. Cao, et al., Carbon quantum dots/TiO composites for efficient photocatalytic hydrogen evolution, J. Mater. Chem. A. 2 (2014) 33443351.

[2] H. Yu, R. Shi, Y. Zhao, G.I. Waterhouse, L.-Z. Wu, C.-H. Tung, et al., Smart utilization of carbon dots in semiconductor photocatalysis, Adv. Mater. 28 (2016) 9454-9477.

[3] W. Zhou, J. Jia, J. Lu, L. Yang, D. Hou, G. Li, et al., Recent developments of carbon-based electrocatalysts for hydrogen evolution reaction, Nano Energy. 28 (2016) 29-43.

[4] N. Briggs, S. Subramanian, Z. Lin, X. Li, X. Zhang, K. Zhang, et al., A roadmap for electronic grade 2D materials, 2D Materials. 6 (2019) 022001.

[5] E. Quesnel, F. Roux, F. Emieux, P. Faucherand, E. Kymakis, G. Volonakis, et al., Graphene-based technologies for energy applications, challenges and perspectives, 2D Materials. 2 (2015) 030204.

[6] Z.W. Seh, J. Kibsgaard, C.F. Dickens, I. Chorkendorff, J.K. Nørskov, T.F. Jaramillo, Combining theory and experiment in electrocatalysis: Insights into materials design, Science. 355 (2017) 4998.

[7] G. Williams, B. Seger, P.V. Kamat, $\mathrm{TiO}_{2}-$ Graphene Nanocomposites. UV-Assisted Photocatalytic Reduction of Graphene Oxide, ACS Nano. 2 (2008) 1487-1491. doi:10.1021/nn800251f.

[8] X. Xie, N. Zhang, Z.-R. Tang, Y.-J. Xu, An adaptive geometry regulation strategy for 3D 
graphene materials: towards advanced hybrid photocatalysts, Chemical Science. 9 (2018) 88768882.

[9] X. An, Jimmy C Yu, Y. Wang, Y. Hu, X. Yu, G. Zhang, $\mathrm{WO}_{3}$ nanorods/graphene nanocomposites for high-efficiency visible-light-driven photocatalysis and $\mathrm{NO}_{2}$ gas sensing, $\mathrm{J}$. Mater. Chem. 22 (2012) 8525-8531.

[10] J. Guo, Y. Li, S. Zhu, Z. Chen, Q. Liu, D. Zhang, et al., Synthesis of $\mathrm{WO}_{3} /$ Graphene composite for enhanced photocatalytic oxygen evolution from water, RSC Adv. 2 (2012) 13561363.

[11] T. Xu, L. Zhang, H. Cheng, Y. Zhu, Significantly enhanced photocatalytic performance of $\mathrm{ZnO}$ via graphene hybridization and the mechanism study, Applied Catalysis B: Environmental. 101 (2011) 382-387.

[12] G. Liao, S. Chen, X. Quan, H. Yu, H. Zhao, Graphene oxide modified $\mathrm{gC}_{3} \mathrm{~N}_{4}$ hybrid with enhanced photocatalytic capability under visible light irradiation, J. Mater. Chem. 22 (2012) 27212726.

[13] Q. Li, X. Li, S. Wageh, A.A. Al Ghamdi, J. Yu, CdS/graphene nanocomposite photocatalysts, Advanced Energy Materials. 5 (2015) 1500010.

[14] Q. Xiang, J. Yu, M. Jaroniec, Graphene-based semiconductor photocatalysts, Chemical Society Review. 41 (2012) 782-796.

[15] J.S. Lee, K.H. You, C.B. Park, Highly Photoactive, Low Bandgap $\mathrm{TiO}_{2}$ Nanoparticles Wrapped by Graphene, Adv. Mater. 24 (2012) 1084-1088.

[16] X. An, J.C. Yu, Graphene-based photocatalytic composites, RSC Adv. 1 (2011) 1426-1434.

[17] N. Zhang, Y. Zhang, Y.-J. Xu, Recent progress on graphene-based photocatalysts: current status and future perspectives, Nanoscale. 4 (2012) 5792-5813.

[18] M. Aqeel, S. Anjum, M. Imran, M. Ikram, H. Majeed, M. Naz, et al., TiO ${ }_{2} @$ RGO (reduced graphene oxide) doped nanoparticles demonstrated improved photocatalytic activity, Materials Research Express. 6 (2019) 086215. 
[19] H. Bin Wu, H.H. Hng, X.W.D. Lou, Direct Synthesis of Anatase $\mathrm{TiO}_{2}$ Nanowires with Enhanced Photocatalytic Activity, Adv. Mater. 24 (2012) 2567-2571.

[20] Y. Zhao, N. Hoivik, K. Wang, Recent advance on engineering titanium dioxide nanotubes for photochemical and photoelectrochemical water splitting, Nano Energy. 30 (2016) 728-744.

[21] T.-F. Yeh, J.M. Syu, C. Cheng, T.H. Chang, H. Teng, Graphite Oxide as a Photocatalyst for Hydrogen Production from Water, Adv. Funct. Mater. 20 (2010) 2255-2262.

[22] H. Huang, S. Tu, C. Zeng, T Zhang, A.H. Reshak, and Y. Zhang, Macroscopic polarization enhancement promoting photo-and piezoelectric-induced charge separation and molecular oxygen activation, Angew. Chem. Int. Ed. 56 (2017) 11860-11864.

[23] L. Hao, L. Kang, H. Huang, L. Ye, K. Han, S. Yang, H. Yu, M. Batmunkh, Y. Zhang, T. Ma, Surface-halogenation-induced atomic-site activation and local charge separation for superb $\mathrm{CO}_{2}$ photoreduction, Adv. Mater. 31 (2019) 1900546.

[24] F. Chen, H. Huang, L. Guo, Y. Zhang, T. Ma, The role of polarization in photocatalysis, Angew. Chem. Int. Ed. 58 (2019) 10061-10073

[25] F. Chen, H. Huang, L. Ye, T. Zhang, Y. Zhang, X. Han, T. Ma, Thickness-dependent facet junction control of layered $\mathrm{BiOIO}_{3}$ single crystals for highly efficient $\mathrm{CO}_{2}$ photoreduction, $\mathrm{Adv}$. Funct. Mater. 46 (2018) 1804284

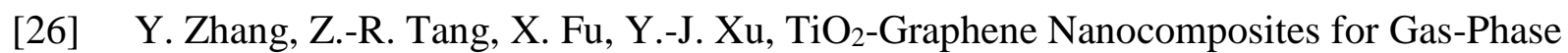
Photocatalytic Degradation of Volatile Aromatic Pollutant: Is $\mathrm{TiO}_{2}-\mathrm{Graphene}$ Truly Different from Other $\mathrm{TiO}_{2}$-Carbon Composite Materials? ACS Nano. 4 (2010) 7303-7314.

[27] J. Wintterlin, M-L. Bocquet, Graphene on metal surfaces, Surf. Sci. 603 (2009) 1841-1852.

[28] M. Batzill, The surface science of graphene: Metal interfaces, CVD synthesis, nanoribbons, chemical modifications, and defects, Surf. Sci. Rep. 67 (2012) 83-115.

[29] C. Riedl, C. Coletti, T. Iwasaki, A.A. Zakharov, U. Starke, Quasi-Free-Standing Epitaxial Graphene on SiC Obtained by Hydrogen Intercalation, Phys. Rev. Lett. 103 (2009) 246804. 
Interaction by Reactive Intercalation, J. Am. Chem. Soc. 132 (2010) 8175-8179.

[31] R. Larciprete, S. Ulstrup, P. Lacovig, M. Dalmiglio, M. Bianchi, F. Mazzola, et al., Oxygen Switching of the Epitaxial Graphene-Metal Interaction, ACS Nano. 6 (2012) 9551-9558.

[32] I. Palacio, G. Otero-Irurueta, Concepció. Alonso, José.I. Martínez, E. López-Elvira, I. MuñozOchando, H.J. Salavagione, Marí.F. López, M. García-Hernández, J. Méndez, G.J.Ellis, José.A. Martín-Gago, Chemistry below graphene: Decoupling epitaxial graphene from metals bypotentialcontrolled electrochemical oxidation, Carbon 129 (2018) 837-846.

[33] R. Mu, Q. Fu, L. Jin, L. Yu, G. Fang, D. Tan, et al., Visualizing Chemical Reactions Confined under Graphene, Angew. Chem. 124 (2012) 4940-4943.

[34] M. Petrović, I.Š. Rakić, S. Runte, C. Busse, J.T. Sadowski, P. Lazić, et al., The mechanism of caesium intercalation of graphene, Nature Communications. 4 (2013) 2772.

[35] C. Enderlein, Y.S. Kim, A. Bostwick, E. Rotenberg, K. Horn, The formation of an energy gap in graphene on ruthenium by controlling the interface, New J. Phys. 12 (2010) 033014.

[36] F. Presel, N. Jabeen, M. Pozzo, D. Curcio, L. Omiciuolo, P. Lacovig, et al., Unravelling the roles of surface chemical composition and geometry for the graphene-metal interaction through $\mathrm{C} 1 \mathrm{~s}$ core-level spectroscopy, Carbon. 93 (2015) 187-198.

[37] L. Omiciuolo, E.R. Hernández, E. Miniussi, F. Orlando, P. Lacovig, S. Lizzit, et al., Bottom-up approach for the low-cost synthesis of graphene-alumina nanosheet interfaces using bimetallic alloys, Nature Communications. 5 (2014) 5062.

[38] S. Lizzit, R. Larciprete, P. Lacovig, M. Dalmiglio, F. Orlando, A. Baraldi, et al., TransferFree Electrical Insulation of Epitaxial Graphene from its Metal Substrate, Nano Lett. 12 (2012) $4503-4507$.

[39] H.-J. Freund, G. Pacchioni, Oxide ultra-thin films on metals: new materials for the design of supported metal catalysts, Chem. Soc. Rev. 37 (2008) 2224. 
[40] J. Azadmanjiri, V.K. Srivastava, P. Kumar, J. Wang, A. Yu, Graphene-supported 2D transition metal oxide heterostructures, J. Mater. Chem. A. 6 (2018) 13509-13537.

[41] P. Das, Q. Fu, X. Bao, Z.-S. Wu, Recent advances in the preparation, characterization, and applications of two-dimensional heterostructures for energy storage and conversion, J. Mater. Chem. A. 6 (2018) 21747-21784.

[42] J. Coraux, A.T.N. Diaye, C. Busse, T. Michely, Structural Coherency of Graphene on $\operatorname{Ir}(111)$, Nano Lett. 8 (2008) 565-570.

[43] H. Tetlow, J. Posthuma De Boer, I.J. Ford, D.D. Vvedensky, J. Coraux, L. Kantorovich, Growth of epitaxial graphene: Theory and experiment, Phys. Rep. 542 (2014) 195.

[44] R.T. Frederick, Z. Novotný, F.P. Netzer, G.S. Herman, Z. Dohnálek, Growth and Stability of Titanium Dioxide Nanoclusters on Graphene/Ru(0001), J. Phys. Chem. B. 122 (2017) 640-648. [45] T. Mashoff, M. Takamura, S. Tanabe, H. Hibino, F. Beltram, S. Heun, Hydrogen storage with titanium-functionalized graphene, Appl. Phys. Lett. 103 (2013) 013903. doi:10.1063/1.4812830.

[46] R. Ruus, A. Kikas, A. Saar, A. Ausmees, E. Nõmmiste, J. Aarik, et al., Ti 2p and O 1s X-ray absorption of $\mathrm{TiO}_{2}$ polymorphs, Solid State Commun. 104 (1997) 199-203.

[47] U. Diebold, N. Ruzycki, G.S. Herman, A. Selloni, One step towards bridging the materials gap: surface studies of $\mathrm{TiO}_{2}$ anatase, Catal. Today. 85 (2003) 93-100.

[48] M.R. Ranade, A. Navrotsky, H.Z. Zhang, J.F. Banfield, S.H. Elder, A. Zaban, et al., Energetics of nanocrystalline $\mathrm{TiO}_{2}$, Proceedings of the National Academy of Sciences of the United States of America. 99 (2002) 6476-6481.

[49] M.C. Biesinger, B.P. Payne, A.P. Grosvenor, L.W.M. Lau, A.R. Gerson, R.S.C. Smart, Resolving surface chemical states in XPS analysis of first row transition metals, oxides and hydroxides: Cr, Mn, Fe, Co and Ni, Appl. Surf. Sci. 257 (2011) 2717-2730.

[50] G. Barcaro, S. Agnoli, F. Sedona, G.A. Rizzi, A. Fortunelli, G. Granozzi, Structure of Reduced Ultrathin $\mathrm{TiO}_{x}$ Polar Films on Pt(111), J. Phys. Chem. C. 113 (2009) 5721-5729. 
[51] K.C. Prince, V.R. Dhanak, P. Finetti, J.F. Walsh, R. Davis, C.A. Muryn, et al., 2p resonant photoemission study of $\mathrm{TiO}_{2}$, Phys. Rev. B. 55 (1997) 9520-9523.

[52] C. Das, M. Richter, M. Tallarida, D. Schmeisser, Electronic properties of atomic layer deposition films, anatase and rutile $\mathrm{TiO}_{2}$ studied by resonant photoemission spectroscopy, J. Phys. D: Appl. Phys. 49 (2016) 275304.

[53] A. Dahal, M. Batzill, Growth from behind: Intercalation-growth of two-dimensional FeO moiré structure underneath of metal-supported graphene, Sci. Rep. 5 (2015) 11378.

[54] I. Pletikosić, M. Kralj, P. Pervan, R. Brako, J. Coraux, A.T. N’Diaye, et al., Dirac Cones and Minigaps for Graphene on $\operatorname{Ir}(111)$, Phys. Rev. Lett. 102 (2009) 056808.

[55] R. Balog, B. Jørgensen, L. Nilsson, M. Andersen, E. Rienks, M. Bianchi, M. Fanetti, E. Lægsgaard, A. Baraldi, S. Lizzit, Z. Sljivancanin, F. Besenbacher, B. Hammer, T.G. Pedersen, P. Hofmann, L. Hornekær, Bandgap opening in graphene induced by patterned hydrogen adsorption, Nat. Mater. 9 (2010) 315-319.

[56] R. Addou, A. Dahal, M. Batzill, Growth of a two-dimensional dielectric monolayer on quasi-freestanding graphene, Nat. Nanotechnol. 8 (2012) 41-45.

[57] A.L. Hsu, R.J. Koch, M.T. Ong, W. Fang, M. Hofmann, K.K. Kim, et al., Surface-Induced Hybridization between Graphene and Titanium, ACS Nano. 8 (2014) 7704-7713.

[58] A.B. Preobrajenski, M.L. Ng, A.S. Vinogradov, N. Mårtensson, Controlling graphene corrugation on lattice-mismatched substrates, Phys. Rev. B. 78 (2008) 073401.

[59] E. Miniussi, M. Pozzo, A. Baraldi, E. Vesselli, R.R. Zhan, G. Comelli, et al., Thermal Stability of Corrugated Epitaxial Graphene Grown on Re(0001), Phys. Rev. Lett. 106 (2011) 216101.

[60] M. Lazzeri, A. Vittadini, A. Selloni, Structure and energetics of stoichiometric $\mathrm{TiO}_{2}$ anatase surfaces, Phys. Rev. B. 63 (2001) 155409.

[61] S. Selcuk, A. Selloni, Facet-dependent trapping and dynamics of excess electrons at anatase $\mathrm{TiO}_{2}$ surfaces and aqueous interfaces, Nat. Mater. 15 (2016) 1107-1112. 
[62] X.-Q. Gong, A. Selloni, M. Batzill, U. Diebold, Steps on anatase $\mathrm{TiO}_{2}(101)$, Nat. Mater. 5 (2006) 665-670.

[63] M. Lazzeri, A. Selloni, Stress-Driven Reconstruction of an Oxide Surface: The Anatase $\mathrm{TiO}_{2}(001)$-(14) Surface, Phys. Rev. Lett. 87 (2001) 266105.

[64] H. Tang, F. Lévy, H. Berger, P.E. Schmid, Urbach tail of anatase $\mathrm{TiO}_{2}$, Phys. Rev. B. 52 (1995) 7771-7774.

[65] M. Bianchi, D. Cassese, A. Cavallin, R. Comin, F. Orlando, L. Postregna, et al., Surface core level shifts of clean and oxygen covered Ir(111), New J. Phys. 11 (2009) 063002.

[66] R. Larciprete, P. Lacovig, F. Orlando, M. Dalmiglio, L. Omiciuolo, A. Baraldi, S. Lizzit, Chemical gating of epitaxial graphene through ultrathin oxide layers, Nanoscale 7 (2015) 12650.

[67] S. Doniach, M. Sunjic, Many-electron singularity in X-ray photoemission and X-ray line spectra from metals, J. Phys. C: Solid State Phys. 3 (1970) 285-291.

[68] D. Nečas, P. Klapetek, Gwyddion: an open-source software for SPM data analysis, Central European Journal of Physics. 10 (2012) 181-188.

[69] G. Kresse, J. Furthmüller, Efficient iterative schemes for ab-initio total-energy calculations using a plane-wave basis set, Phys. Rev. B. 54 (1996) 11169-11186.

[70] I. Hamada, van der Waals density functional made accurate, Phys. Rev. B. 89 (2014) 121103.

[71] P.E. Blöchl, Projector augmented-wave method, Phys. Rev. B. 50 (1994) 17953-17979.

[72] J.P. Perdew, K. Burke, M. Ernzerhof, Generalized Gradient Approximation Made Simple, Phys. Rev. Lett. 77 (1996) 3865-3868. 
[73] P.N.O. Gillespie, N. Martsinovich, Electronic Structure and Charge Transfer in the $\mathrm{TiO}_{2}$ Rutile (110)/Graphene Composite Using Hybrid DFT Calculations, J. Phys. Chem. C. 121 (2017) $4158-4171$.

[74] J. Heyd, G.E. Scuseria, M. Ernzerhof, Hybrid functionals based on a screened Coulomb potential, J. Chem. Phys. 118 (2003) 8207-8215.

[75] D.J. Chadi, Special points for Brillouin-zone integrations, Phys. Rev. B. 16 (1977) 17461747. 